\title{
On 3-Layer Crossings and Pseudo Arrangements
}

\author{
Farhad Shahrokhi ${ }^{1 \star}$ and Imrich Vrto ${ }^{2 \star \star}$ \\ 1 Department of Computer Science, University of North Texas \\ P.O.Box 13886, Denton, TX, USA \\ farhad@csci.unt.edu \\ 2 Department of Informatics, Institute of Mathematics \\ Slovak Academy of Sciences \\ P.O.Box 56, 84000 Bratislava, Slovak Republic \\ vrto@savba.sk
}

\begin{abstract}
Let $G=\left(V_{0}, V_{1}, V_{2}, E\right)$ be a 3-layer graph. The 3-layer drawings of $G$ in which $V_{0}, V_{1}$, and $V_{2}$ are placed on 3 parallel lines and each edge in $E$ is drawn using one straight line segment, are studied. A generalization of the linear arrangement problem which we call the 3layer pseudo linear arrangement problem is introduced, and it is shown to be closely related to the 3-layer crossing number. In particular, we show that the 3-layer crossing number of $G$ plus the sum of the square of degrees asymptotically has the same order of magnitude as the optimal solution to the 3-layer linear arrangement problem. Consequently, when $G$ satisfies certain (reasonable) assumptions, we derive the first polynomial time approximation algorithm to compute the 3-layer crossing number within a multiplicative factor of $O(\log n)$ from the optimal.
\end{abstract}

\section{Introduction}

The planar crossing number problem is the problem of placing the vertices of a graph in the plane and drawing the edges with curves, to minimize the number of edge crossings [20]. This problem is known to be NP-hard [9] and has been extensively studied in graph theory [23,16], and theory of VLSI [13]. One of the most important aesthetic objectives in drawing graphs is to have a small number of crossings [17], and therefore the crossing minimization problems have been frequently studied by the graph drawing community e.g. $[4,5,11,15]$.

Let $G=(V, E)$ be an undirected graph with the vertex set $V$ and the edge set $E . G$ is called a $k$-layer graph, if a partition of $V$ into $k$ sets $V_{0}, V_{1}, \ldots, V_{k-1}$ exists so that any edge in $E$ has one end point in $V_{i}$ and the other end point in $V_{i+1}$ for some $i=0,1,2, \ldots, k-2$. Thus, any bipartite graph is a 2-layer graph. If $G=(V, E)$ is a $k$-layer graph, then we write $G=\left(V_{0}, V_{1}, \ldots, V_{k-1}, E\right)$, where $\left\{V_{0}, V_{1}, \ldots, V_{k-1}\right\}$ is the partition of $V$ into $k$ disjoint sets. Let $G=$

\footnotetext{
* This research was supported by NSF grant CCR-9528228. A part of this work was completed when the first author was visiting DIMACS in the Fall of 1998.

** This research was supported by grant No. 95/5305/277 of The Slovak Grant Agency.
} 
$\left(V_{0}, V_{2}, \ldots, V_{k-1}, E\right)$ be a $k$-layer graph, $k \geq 2$, and let $L_{0}, L_{1}, \ldots, L_{k-1}$ be $k$ parallel lines in the plane. A $k$-layer drawing $[1,4,12,15,21]$ of $G$ consists of placing the vertices of $V_{i}$ into distinct points on $L_{i}, i=0,1,2 \ldots, k-1$, and then drawing each edge using a straight line segment connecting the points representing the end vertices of the edge. The objective is to minimize the number of crossings between the edge pairs. Note that any $k$-layer drawing of $G$ is identified by a one to one function

$$
D: V_{0} \cup V_{1} \ldots \cup V_{k-1} \rightarrow \Re
$$

where $\Re$ is the set of non-negative real numbers. In particular, note that the restriction of $D$ to $V_{i}, 0 \leq i \leq k-1$, specifies the order in which the vertices of $V_{i}$ will appear on $L_{i}$. When $k=2$ the corresponding drawing is called a bipartite drawing and the problem of minimizing the number of crossings is called the bipartite crossing number problem $[5,11,15,21,19]$.

Computing the bipartite crossing number is NP-hard $[9]^{1}$ and despite a great deal of research which was done on this problem, no polynomial time approximation algorithm had been known for this problem. Very recently a polynomial time approximation algorithm with performance guarantee of $O(\log n)$ from the optimal was discovered for approximating the bipartite crossing number of a large class of graphs on $n$ vertices [19]. Nonetheless, no efficient approximation algorithm for minimizing the number of crossings in a $k$-layer drawing has been known for $k \geq 3$. The main results in [19] were obtained by relating the bipartite crossing number problem to the linear arrangement problem which is another well known problem in theory of VLSI. In this paper we develop a general framework to study the 3-layer crossing number problem by relating it to a very general version of the linear arrangement problem which we call the pseudoarrangement problem. In particular, we derive tight upper and lower bounds for the 3-layer crossing number in terms of the arrangement values. The ratio of the main term of the upper bound to the main term in the lower bound is only 3 , and the error term is the sum of the square of degrees in $G$. The result is interesting, since it indicates that the number of edge crossings is closely related to the length of the drawing which is defined by the pseudo-arrangement problem. Consequently, we derive the first polynomial time approximation algorithm for the 3-layer crossing problem with the performance guarantee of $O(\log n)$ from the optimal, provided that the graph satisfies certain conditions.

\section{Basic Concepts and Notations}

Let $G=(V, E)$ be a graph, we denote by $d_{v}$ the degree of $v \in V$. Throughout this paper $G=(A, B, E)$ denotes a bipartite graph on the partite sets $A$ and $B$, and the edge set $E$. $G=\left(V_{0}, V_{1}, V_{2}, E\right)$ denotes a 3-layer graph, with vertex set $V=V_{0} \cup V_{1} \cup V_{2},|V|=n$, and the edge set $E$.

${ }_{1}$ Technically speaking, the NP-hardness of the problem was proved for multigraphs, but it is widely assumed that it is also NP-hard for simple graphs. 
For $G=\left(V_{0}, V_{1}, V_{2}, E\right)$, let $G_{0}$ and $G_{2}$ denote, respectively, the induced subgraphs of $G$ on the vertex sets $V_{0} \cup V_{1}$, and $V_{2} \cup V_{1}$. Note that $G_{0}$ and $G_{2}$ are bipartite graphs. Note that there is no edge in $G$ with one end vertex in $V_{0}$ and the other in $V_{2}$. For any $v \in V_{1}$, let $d_{v, 0}$ and $d_{v, 2}$ denote the degrees of $v$, in $G_{0}$ and $G_{2}$, respectively, and note that $d_{v, 0}+d_{v, 2}=d_{v}$.

A 3-layer drawing of $G=\left(V_{0}, V_{1}, V_{2}\right)$ is a one-to-one function

$$
D: V_{0} \cup V_{1} \cup V_{2} \rightarrow \Re .
$$

Let $D_{i}$ denote the restriction of $D$ to $V_{i}, 0 \leq i \leq 2$. Note that $D_{i}, 0 \leq i \leq 2$ specifies the order in which the vertices in $V_{i}$ are placed on the line $L_{i}$. Observe that $\left(D_{0}, D_{1}\right)$ is a bipartite drawing for $G_{0}$ and $\left(D_{1}, D_{2}\right)$ is a bipartite drawing for $G_{2}$. For any $e \in E$, let $c r_{0}(e)$ denote the number of crossings of $e$ with other edges in the drawing $\left(D_{0}, D_{1}\right)$, and $\mathrm{cr}_{2}(e)$ denote the number of crossings of $e$ with other edges in the drawing $\left(D_{1}, D_{2}\right)$. We define $c r_{0}$ and $c r_{2}$ to be the total number of crossings in the drawings $\left(D_{0}, D_{1}\right)$ and $\left(D_{1}, D_{2}\right)$, respectively, and define $c r_{D}$ to be the total number of edge crossings in $D$. Thus, $c r_{D}=c r_{0}+c r_{2}$. The 3-layer crossing number of $G$ is the minimum number of crossings of edges over all 3-layer drawings of $G$.

A linear arrangement (LA) of a graph $G=(V, E)$ is a one to one function $f: V \rightarrow\{1,2, \ldots|V|\}$. The linear arrangement problem is to find a LA so that $\sum_{u v \in E}|f(u)-f(v)|$ is minimized $[2,3,7,10,18]$. This problem is known to be NP-hard but can be approximated in polynomial time using a variety of algorithms $[7,10,18]$. Crucial to our work are generalizations of this problem defined for bipartite and 3-layer graphs.

For $x, y \in \Re$, let $(x, y)$ denote the open interval between $x$ and $y$. Let $G=$ $(A, B, E)$. A pseudo linear arrangement (PLA) for $G$ is a one to one function $f: A \cup B \rightarrow \Re$ so that $f(B)=\{1,2 \ldots,|B|\}$. Hence, any vertex in $B$ is assigned a unique integer which is at most equal to $|B|$. Let $a b \in E$, with $f(a)<f(b)$. We define the length of $e$, denoted by $L_{f}^{e}$, to be

$$
\sum_{x \in B, f(x) \in(f(a), f(b))} d_{x}
$$

We define the length of $f$, denoted by $L_{f}$, to be $\sum_{a b \in E} L_{f}^{e}$. The pseudo linear arrangement problem is to find a PLA of minimum length. We denote this minimum value by $\bar{L}_{G}$. It follows from the recent work on spreading matrices $[7,18]$ that for any graph on $n$ vertices $\bar{L}_{G}$ can be approximated to within a factor of $O(\log n)$ from the optimal in polynomial time.

A 3-layer pseudo linear arrangement (3PLA) of $G=\left(V_{0}, V_{1}, V_{2}, E\right)$ is a one to one function $f: V_{0} \cup V_{1} \cup V_{2} \rightarrow \Re$, so that $f\left(V_{1}\right)=\left\{1,2,3, \ldots,\left|V_{1}\right|\right\}$. Note that we may view any 3PLA $f$ of $G$ as a 3-layer drawing of $G$. Let $f_{i}$ denote the restriction of $f$ to $V_{i}, 0 \leq i \leq 2$, and note that that $\left(f_{0}, f_{1}\right)$ and $\left(f_{1}, f_{2}\right)$ are PLAs of $G_{0}$ and $G_{2}$, respectively. Let $a b \in E$, we define the length of $e$, denoted by $L_{f}^{e}$ to be $L_{\left(f_{0}, f_{1}\right)}^{e}$, provided that $e$ is an edge in $G_{0}$, otherwise, we define $L_{f}^{e}$ to be $L_{\left(f_{1}, f_{2}\right)}^{e}$. Note that for any edge $e$ in $G_{0}$ 


$$
L_{f}^{e}=\sum_{x \in V_{1}, f(x) \in(f(a), f(b))} d_{x, 0}
$$

whereas, for any $e$ in $G_{2}$,

$$
L_{f}^{e}=\sum_{x \in V_{1}, f(x) \in(f(a), f(b))} d_{x, 2} .
$$

The length of $f$, denoted by $L_{f}$, is defined to be $\sum_{e \in E} L_{f}^{e}$. The 3-layer pseudo linear arrangement problem is to find a 3PLA for $G$ which has the minimum length. We denote this minimum value by $L_{G}$. Let $f$ be a 3 PLA of $G$. For $v \in V_{0} \cup V_{2}$, let $u_{1}, u_{2}, \ldots, u_{d_{v}}$ be its neighbors in the set $V_{1}$ satisfying $f\left(u_{1}\right)<$ $f\left(u_{2}\right)<\ldots<f\left(u_{d_{v}}\right)$. We define the median vertex of $v$, denoted by $\operatorname{med}(v)$, to be $u_{\left\lceil\frac{d_{v}}{2}\right\rceil}$.

\section{Arrangements and 3-Layer Drawings}

Let $G=\left(V_{0}, V_{1}, V_{2}, E\right)$ and $D$ be a 3-layer drawing of $G$. We assume throughout this paper that the vertices of $V_{0}$ are placed on the line $y=0$, vertices of $V_{1}$ are placed on the line, $y=1$, and vertices of $V_{3}$ are placed on the line $y=2$. Moreover, since the number of crossings only depends on the order of vertices, we will assume throughout this paper that the vertices of $V_{1}$ are placed into the points

$$
(1,1),(2,1), \ldots,\left(\left|V_{1}\right|, 1\right)
$$

Note that for any $v \in V, D(v)$ is the $x$-coordinate of $v$, and that $D$ is a 3PLA of $G$. In particular, note that for any $v \in V_{0} \cup V_{2}, \operatorname{med}(v)$ is the vertex $u_{\left\lceil\frac{d_{v}}{2}\right\rceil}$, where $u_{1}, u_{2}, \ldots, u_{d_{v}}$ are neighbors of $v$ in the set $V_{1}$ satisfying $D\left(u_{1}\right)<D\left(u_{2}\right)<$ $\ldots<D\left(u_{d_{v}}\right)$.

Theorem 1. Let $D$ be a 3-layer drawing of $G=\left(V_{0}, V_{1}, V_{2}, E\right)$, then

$$
c r_{D} \geq \frac{1}{2}\left(L_{G}-\sum_{v \in V_{0} \cup V_{2}}\left\lfloor\frac{d_{v}}{2}\right\rfloor d_{v}\right)
$$

Proof. To show the lower bound on $c r_{D}$, consider the bipartite drawing $\left(D_{0}, D_{1}\right)$ of $G_{0}$. Let $v \in V_{0}$ with $d_{v} \geq 2$, and let $u_{1}, u_{2}, \ldots, u_{d_{v}}$ be its neighbors with $D\left(u_{1}\right)<D\left(u_{2}\right)<\ldots<D\left(u_{d_{v}}\right)$. Let $i$ be an integer, $1 \leq i \leq\left\lfloor d_{v} / 2\right\rfloor$, and let $u$ be a vertex in $V_{1}$ so that that $D\left(u_{i}\right)<D(u)<D\left(u_{d_{v}-i+1}\right)$. Observe that $u$ generates $d_{u, 0}$ crossings on the edges $u_{i} v$ and $u_{d_{v}-i+1} v$, if it is not adjacent to $v$; similarly, $u$ generates $d_{u, 0}-1$ crossings on the edges $u_{i} v$ and $u_{d_{v}-i+1} v$, if it is adjacent to $v$. Thus,

$$
c r_{0}\left(u_{i} v\right)+c r_{0}\left(u_{d_{v}-i+1} v\right) \geq \sum_{D(u) \in\left(D\left(u_{i}\right), D\left(u_{d_{v}-i+1}\right)\right)} d_{u, 0}-d_{v} .
$$


Hence, for $v \in V_{0}$ with $d_{v} \geq 2$,

$$
\sum_{i=1}^{d_{v}} c r_{D_{0}}\left(u_{i} v\right) \geq \sum_{i=1}^{\left\lfloor\frac{d_{v}}{2}\right\rfloor} \sum_{D(u) \in\left(D\left(u_{i}\right), D\left(u_{d_{v}-i+1}\right)\right)} d_{u, 0}-\left\lfloor\frac{d_{v}}{2}\right\rfloor d_{v}
$$

We conclude by taking the sum over all $v \in V_{0}, d_{v} \geq 2$ that,

$$
2 c r_{0} \geq \sum_{v \in V_{0}} \sum_{i=1}^{d_{v}} c r_{0}\left(u_{i} v\right) \geq \sum_{v \in V_{0}} \sum_{i=1}^{\left\lfloor\frac{d_{v}}{2}\right\rfloor} \sum_{D(u) \in\left(D\left(u_{i}\right), D\left(u_{\left.d_{v}-i+1\right)}\right)\right.} d_{u, 0}-\sum_{v \in V_{0}}\left\lfloor\frac{d_{v}}{2}\right\rfloor d_{v} .
$$

Using a similar approach we obtain

$$
2 c r_{2} \geq \sum_{v \in V_{2}} \sum_{i=1}^{d_{v}} c r_{2}\left(u_{i} v\right) \geq \sum_{v \in V_{2}} \sum_{i=1}^{\left\lfloor\frac{d_{v}}{2}\right\rfloor} \sum_{D(u) \in\left(D\left(u_{i}\right), D\left(u_{d_{v}-i+1}\right)\right)} d_{2,0}-\sum_{v \in V_{2}}\left\lfloor\frac{d_{v}}{2}\right\rfloor d_{v} .
$$

Define a 3PLA by: $f(v)=D(v)$ for all $v \in V_{1}$, and $f(v)=\operatorname{med}(v)+\epsilon_{v}$ for all $v \in V_{0} \cup V_{2}$, where $\epsilon_{v}$ is an infinitely small value. Let $e=v x$, where $v \in V_{0} \cup V_{2}$, and $x=\operatorname{med}(v)$, then $L_{f}^{e}=0$. It follows that

$$
L_{f}=\sum_{v \in V_{0} \cup V_{2}} \sum_{i=1}^{\left\lfloor\frac{\left.d_{v}\right\rfloor}{2}\right\rfloor} \sum_{D(u) \in\left(D\left(u_{i}\right), D\left(u_{d_{v}-i+1}\right)\right)} d_{u, 0}
$$

Hence, $2 c r_{D} \geq L_{f}-\sum_{v \in V_{0} \cup V_{2}}\left\lfloor\frac{d_{v}}{2}\right\rfloor d_{v}$, and the claim follows by observing that $L_{G} \leq L_{f}$.

Theorem 2. Let $f$ be a $3 P L A$ of $G=\left(V_{0}, V_{1}, V_{2}, E\right)$, then there is 3-layer drawing $D$ of $G$ so that

$$
c r_{D} \leq \frac{3}{2} L_{f}+\sum_{v \in V_{1}} d_{v}^{2}
$$

Proof. Define a new 3PLA by: $D(v)=f(\operatorname{med}(v))$ for any $v \in V_{0} \cup V_{2}$, and $D(v)=f(v)$, for any $v \in V_{1}$. If two vertices are placed at the same location, we separate them by placing an arbitrary small distance between them.

$$
L_{D} \leq L_{f}
$$

To prove the upper bound on $c r_{D}$, we estimate from above the number of crossings on any edge incident to a vertex $v \in V_{0} \cup V_{2}$. The sum of the number of crossings can be shown to be at most $3 / 2 L_{D}+\sum_{v \in V_{1}} d_{v}^{2}$ using the method to derive the lower bound.

By Theorems 1 and 2, in order to construct a 3-layer drawing with small number of crossings, one only needs to find a 3PLA of $G$ with small value. Unfortunately, it is not known how to compute exactly or even approximate 
the 3-layer arrangement problem, in polynomial time. Nonetheless, we can show that if $G_{0}$ and $G_{2}$ do not have vertices of degree zero, then, this problem "essentially" becomes the pseudo linear arrangement problem, provided that $G$ is degree bounded. Let $G=\left(V_{0}, V_{1}, V_{2}, E\right)$, we denote the maximum degree among all vertices in $V_{1}$ by $\Delta_{1}$.

Lemma 1. Let $G=\left(V_{0}, V_{1}, V_{2}, E\right)$, and let $\bar{f}$ be any PLA for the bipartite graph $G=\left(V_{0} \cup V_{2}, V_{1}, E\right)$. Then, $\bar{f}$ induces a $3 P L A$, denoted by $f$, so that

$$
L_{f} \leq L_{\bar{f}}
$$

Moreover, if $d_{v, 0}>0$ and $d_{v, 2}>0$, for any $v \in V_{1}$, then

$$
\frac{L_{\bar{f}}}{\Delta_{1}} \leq L_{f} .
$$

We can now present our main result.

Theorem 3. Let $G=\left(V_{0}, V_{1}, V_{2}, E\right)$ so that $|E|>(2+\epsilon)|V|$, for a positive $\epsilon$. Assume that $d_{v, 0}>0$ and $d_{v, 2}>0$, for all $v \in V_{1}$, and that $\Delta_{1}$ is bounded by a constant. Then, the 3-layer crossing number can be approximated to within a factor of $O(\log n)$ from the optimal in polynomial time.

Proof. The conditions on $E$ and $\Delta_{1}$ can be used to show that for any 3-layer drawing $D$,

$$
c r_{D}=\Omega\left(\sum_{v \in V_{0} \cup V_{2}} d_{v}^{2}\right) .
$$

Hence, by Theorem $1 c r_{D}=\Omega\left(L_{G}\right)$. It remains to construct an $O(\log n)$ times optimal 3PLA, denoted by $f$, since then by Theorem 2 we can construct the desired drawing $D$. To construct $f$, we first construct a $O(\log n)$ times optimal PLA using the algorithm in [18]. By Lemma 1 this gives a $O(\log n)$ times optimal $3 \mathrm{PLA}$, or $f$, since $\Delta_{1}$ is bounded by a constant.

\section{References}

1. Catarci, T.: The assignment heuristics for crossing reduction. IEEE Transactions on Systems, Man and Cybernetics 25 (1995) 515-521

2. Chung, F. R. K.: On optimal linear arrangements of trees. Computers and Mathematics with Applications 10 (1984) 43-60

3. Díaz, J.: Graph layout problems. In International Symposium on Mathematical Foundations of Computer Sciences. Lecture Notes in Computer Science, Vol. 629. Springer-Verlag, Berlin Heidelberg New York (1992) 14-21

4. Di Battista, J., Eades, P., Tamassia, R., Tollis, I. G.: Algorithms for drawing graphs: an annotated bibliography. Computational Geometry 4 (1994) 235-282

5. Eades, P., Wormald, N.: Edge crossings in drawings of bipartite graphs. Algorithmica 11 (1994) 379-403

6. Eades, P., Whitesides, S.: Drawing graphs in 2 layers. Theoretical Computer Science 131 (1994) 361-374 
7. Even, G., Naor, J. S., Rao, S., Schieber, B.: Divide-and-Conquer approximation algorithms via spreading matrices. In 36th Annual IEEE Symposium on Foundation of Computer Science. IEEE Computer Society Press (1995) 62-71

8. Even, G., Naor, J. S., Rao, S., Schieber, B.: Fast approximate graph partition algorithms. In 8th Annual ACM-SIAM Symposium on Discrete Algorithms. ACM Press (1997) 639-648

9. Garey, M. R., Johnson, D. S.: Crossing number is NP-complete. SIAM J. Algebraic and Discrete Methods 4 (1983) 312-316

10. Hansen, M.: Approximate algorithms for geometric embeddings in the plane with applications to parallel processing problems. In 30th Annual IEEE Symposium on Foundation of Computer Science. IEEE Computer Society Press (1989) 604-609

11. Jünger, M., Mutzel, P.: Exact and heuristic algorithm for 2-layer straight line crossing number. In 3rd Symposium on Graph Drawing'95. Lecture Notes in Computer Science, Vol. 1027. Springer-Verlag, BerlinHeidelberg New York (1996) 337-348

12. Jünger, M., Lee, E. K., Mutzel, P., Odenthal T.: A polyhedral approach to the multi-layer crossing number problem. In 5th Symposium on Graph Drawing'97. Lecture Notes in Computer Science, Vol. 1353. Springer-Verlag, Berlin Heidelberg New York (1997) 13-24

13. Leighton, F. T.: Complexity issues in VLSI. MIT Press, Massachusetts (1983)

14. May, M., Szkatula, K.: On the bipartite crossing number. Control and Cybernetics 17 (1988) 85-98

15. Mutzel, P.: An alternative method to crossing minimization on hierarchical graphs. In 4th Symposium on Graph Drawing'96. Lecture Notes in Computer Science, Vol. 1190. Springer-Verlag, Berlin Heidelberg New York (1997) 318-333

16. Pach, J., Agarwal, K.: Combinatorial Geometry. John Wiley \& Sons Inc., New York (1995)

17. Purchase, H.: Which aesthetics has the greatest effect on human understanding? In 5th Symposium on Graph Drawing'97. Lecture Notes in Computer Science, Vol. 1353. Springer-Verlag, Berlin Heidelberg New York (1997) 248-261

18. Rao, S., Richa, A.:, New approximation techniques for some ordering problems. In: 9th Annual ACM-SIAM Symposium on Discrete Algorithms. ACM Press (1998) $211-225$

19. Shahrokhi, F., Sýkora, O., Székely, L. A., Vrto , I.: On bipartite crossings, largest biplanar subgraphs, and the linear arrangement problem. In Workshop on Algorithms and Data Structures'97. Lecture Notes in Computer Science, Vol. 1272. Springer-Verlag, Berlin Heidelberg New York (1997) 55-68

Extended version will appear in SIAM Journal on Computing as: On bipartite drawings and the linear arrangement problem.

20. Shahrokhi, F., Sýkora, O., Székely, L.A., and Vrto , I.: Crossing number problems: bounds and applications. In: Bárány, I., and Böröczky, K. (eds): Intuitive Geometry. Bolyai Society Mathematical Studies, Vol 6. Akadémia Kiadó, Budapest (1997) 179-206

21. Sugiyama, K., Tagawa, S., Toda, M.: Methods for visual understanding of hierarchical systems structures. IEEE Transactions on Systems, Man and Cybernetics 11 (1981) 109-125

22. Warfield, J.: Crossing theory and hierarchy mapping. IEEE Transactions on Systems, Man and Cybernetics 7 (1977) 502-523

23. White, A. T., and Beineke, L. W.: Topological graph theory. In: L. W. Beineke, L. W., and R. J. Wilson, R. J. (eds.): Selected Topics in Graph Theory. Academic Press, New York (1978) 15-50 